\title{
How can we improve the translational landscape for a faster cure of type 1 diabetes?
}

\author{
Matthias von Herrath ${ }^{1}$ and Andrew Chan ${ }^{2}$
}

${ }^{1}$ La Jolla Institute for Allergy and Immunology, La Jolla, California, USA. ${ }^{2}$ Genentech Inc., South San Francisco, California, USA.

\begin{abstract}
Translation of novel therapies for type 1 diabetes and other autoimmune diseases to the clinic has been slow despite significant new initiatives from funding agencies. One reason for this is that different incentives drive industry, academia, and funding bodies. These communities therefore lack common goals and often communicate poorly, resulting in unintended obstacles that hamper progress in efficiently translating basic scientific discoveries into medical practice. Here, based on our own personal experiences, we discuss some of the drivers within each community that cause these problems, existing mechanisms to facilitate the translation of science into medical practice, and remaining issues that need to be solved.
\end{abstract}

\section{The need for change}

Autoimmune diseases in which disruption of normal immunity results in severe end-organ damage include systemic lupus erythematosus, psoriasis, multiple sclerosis, and type 1 diabetes mellitus (T1D) and encompass a wide range of clinical disorders crossing many clinical disciplines $(1,2)$. Most current efforts to develop drugs to treat individuals with these disorders focus primarily on improving clinical symptoms of the diseases and on halting or altering disease progression, because these are FDAapprovable clinical trial endpoints. Typically, symptom-relieving therapies (for example, insulin in T1D) do not correct the underlying immune dysregulation, and systemic immunosuppressants simultaneously compromise normal immune responses against invading pathogens and normal tumor immunosurveillance (3). For most autoimmune disorders, and T1D in particular, developing therapies that correct the underlying pathogenic causes is a tremendous challenge that requires not only meaningful clinical efficacy and long-term benefit to patients, but also extremely safe therapies (4). When this need for a high therapeutic index is coupled with difficulties in predicting clinical success from current preclinical models (mostly inbred mouse strains) and a paucity of biomarkers that reflect disease pathogenesis and progression, the discovery and development of drugs for chronic autoimmune diseases becomes a high-risk commercial enterprise. This is worsened by the low percentage (approximately 20\%) of therapies entering phase I clinical trials that ultimately receive FDA approval (5). In the end, fully burdened costs for developing a single drug from phase I clinical trials to FDA approval are currently estimated to be approximately $\$ 1.2$ billion, with the process taking an estimated 9+ years (5). In addition, in $\mathrm{T} 1 \mathrm{D}$, it is presently apparent that no single drug is likely to prevent or cure the disease and that combinatorial therapeutic approaches are likely to be required. This further complicates the translational landscape because more than one company may be involved and some of the drugs by themselves

Conflict of interest: Matthias von Herrath is a consultant for Novo Nordisk, Baxter, and MannKind and a collaborator with Kyowa Hakko Kirin and Genentech. He receives research funds from Kirin USA.

Nonstandard abbreviations used: IP, intellectual property; JDRF, Juvenile Diabetes Research Foundation; T1D, type 1 diabetes mellitus.

Citation for this article: J. Clin. Invest. 119:1061-1065 (2009). doi:10.1172/JCI37593. may not meet FDA-acceptable endpoints and thus can only be efficient and licensed in conjunction with other drugs.

Some of the hurdles that need to be overcome, if the efficiency of the drug discovery and development process is to be enhanced, stem from shortfalls in the interactions among academia, industry, and funding bodies, which can be improved. Here we review the current translational landscape for preventing or curing T1D; discuss some of the underlying issues that hamper efficient discovery and development of interventions, focusing on the shortfalls in the interactions among academia, industry, and funding bodies; and suggest some improvements that we hope will ignite further discussions that should ultimately lead to a more streamlined process. To better illustrate the underlying issues, we share our own experiences dealing with the hurdles that hamper rapid translation of a combinatorial therapy for T1D.

\section{Academia-industry collaborations: a need for improvement}

The missions and incentives for academia and industry are quite distinct (Figure 1). However, the ability of academia and industry to collaborate in more efficient and productive ways is necessary, if we are to better translate scientific ideas and observations into therapeutics (Figure 1). Each domain brings different resources and skills that are required for ultimate success. Academic resources, which are still mostly driven by single-investigator initiatives, include discovery of new ideas and new biology as well as patient access for observational studies. Major shortfalls are the lack of encouragement for teamwork and the lack of sufficient incentives and stability to build a translational career in biological sciences (recently discussed in detail in ref. 6). Conversely, industry has complementary resources because research and development is structured in larger, team-based efforts. Industry is able to marshal larger amounts of financial, chemical, and technological resources for a single project; can better maneuver the regulatory path to first-in-human studies; can channel both the monetary and personnel resources required to execute large phase II and III clinical trials; and can collect and analyze large numbers of patient samples to explore biomarkers to define patient heterogeneity, disease prognosis, and response to therapies. There are several ongoing efforts toward speeding industry translation of academic advances to human therapeutics, some of which should be expanded and improved in the 


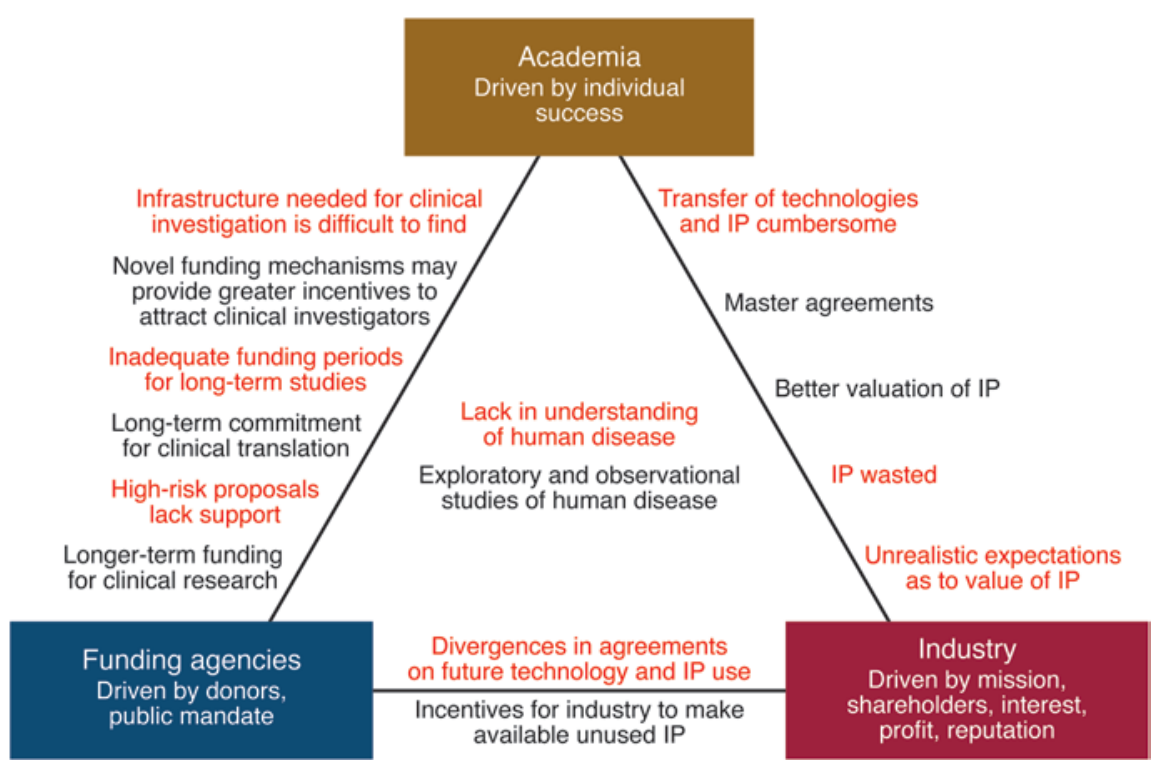

Figure 1

Forces at work in the landscape of translational medicine. It is worthwhile to take a closer look at the driving forces and dynamics that currently govern the translation of basic science into therapeutics for autoimmune disorders. Despite existing synergistic interactions, there are obvious gaps to close, since academia, funding bodies, and industry are motivated by different forces. Closing such gaps could greatly accelerate the development of new biological therapeutics and reduce the time to market for new drugs. Red text indicates antagonistic interests; black text indicates potential solutions.

future. We discuss our view on these here and attempt to make constructive suggestions for further improvement.

\section{Existing mechanisms of collaboration}

Discovery centers. An older, and now less utilized, strategy was for industry to establish discovery centers, where researchers focused on basic scientific discoveries with the hope that the supporting pharmaceutical company could translate some of these discoveries into therapeutic targets. Examples of this approach include the DNAX Research Institute, established by Schering-Plough; the Basel Institute for Immunology and the Roche Institute for Molecular Biology, established by F. Hoffmann-La Roche; and the Hagedorn Research Institute, established by Novo Nordisk. While a handful of discoveries made at such centers are still being tested in clinical trials, this model has been largely abandoned due to low returns on investment (i.e., the centers were too expensive to run and maintain in exchange for the number of new inventions) and the lack of aligned goals and interactions between the basic discovery and drug translational centers.

Industry funding of specific academic researchers and/or projects. Another way in which academia and industry collaborate is for industry to provide project-specific funding for academic investigators to answer product-specific questions as well as for industry to sponsor academic projects for broader biologic questions. In principle, these two approaches are useful collaborations, but rights to existing and new intellectual property (IP) can often complicate negotiations. One solution is to preemptively form agreements between industry and academic institutions that foresee the precise terms of licensing arrangements for new discoveries, as has been recently accomplished by Pfizer with a variety of academic institutions including Washing- ton University, University of California at San Francisco, The Scripps Research Institute, and University of Pennsylvania.

Traditional licensing of academic discoveries. The current paradigm for translating scientific discoveries into therapeutics is the traditional licensing of academic inventions by industry. Once an academic investigator has made a discovery and has established some preliminary IP, their institutional office of technology transfer attempts to license the new invention to corporate partners. While successful in many instances, this process can unfortunately be a rather frustrating process for both sides, with many misunderstandings and misconceptions. For example, because the current academic culture awards individual success, academics tend to overstate the utility of their discoveries and overestimate their usefulness. Many studies are carried out in only one inbred mouse strain, performed in the preventive (rather than interventional) setting for T1D, and not verified in more than one experimental model. The present academic climate also favors publication of positive, but not negative, results, thus leaving out half of the equation needed to rationally translate from bench to bedside. For these and other reasons, industry tends to want full control and to verify/develop new discoveries in-house prior to a licensing agreement or once a licensing agreement has been reached, meaning that the individual investigator, who can be knowledgeable and emotionally invested in the invention, may be cut out of the loop. This could be avoided if negotiations between academic investigators, industry, and legal representation started earlier, so that more mutual understanding on scientific and translational issues could be achieved.

\section{Obstacles to collaboration}

$I P$. At present, academia, industry, and legal representation often work in separate camps. There have been numerous cases in which patent protection was destroyed because academic input was not sought by industry and, conversely, because expert legal help and industry input were not sought by academic institutions. Many technology transfer offices in academic institutions do not have the appropriate resources to build the proper patent portfolios needed to protect a new invention. In addition, if a company does not remain interested in a new technology, technology transfer offices at academic institutions tend to pursue patents and seek out other licensees without seeking much additional input from the investigator. Leadership at institutions, universities, and in industry has to understand the magnitude of this problem and implement structural changes that enforce more frequent and efficient communication. It is instrumental to improve communication between the inventor, interested companies, legal advisors, and technology transfer offices so that IP protection arising from early discoveries can be maximized (Figure 1).

"Fair market value." The gulf between academia and industry in seeking to translate basic scientific discoveries into therapeutics also frequently involves, by necessity, different perspectives on 


\section{Suggested changes to scientific and medical advancement processes}

\section{Academia:}

Reward academic efforts based on contribution to larger teams.

Create a forum for rapid and brief peer-reviewed communication of negative experimental data - this could be web-based and supported by funding bodies such as the NIDDK or JDRF.

Align expectations for the value of IP with industry early on.

Secure core funding for institutes and universities to foster more consistency and cooperation and less competition.

Industry:

Actively educate academia.

Pursue umbrella licensing agreements with academia.

Fulfill humanitarian obligation to pursue orphaned diseases.

Create legal departments that have as a secondary mission the pursuit of improvement of human health, in addition to the protection of IP.

Funding bodies:

Reward and support clinical studies and provide bridging funds (funds to close a crucial temporary gap) for clinical investigators.

Retain the infrastructure created for long-term clinical endeavors on a non-competitive, long-term basis (this still necessitates appropriate oversight for usage of funds), e.g., islet transplantation centers and organ acquisition efforts such as the Network for Pancreatic Organ Donors with Diabetes.

Enforce the use of IP abandoned by industry, to avoid shelving.

Create databases for negative findings that would otherwise remain inaccessible or unpublished.

Facilitate negotiations among the FDA, industry, and academics.

Call to change legislation, if necessary (e.g., extension of patents).

humanitarian versus commercial interests. Industry has to pay heed to investors and market considerations, which are important issues that are frequently not fully appreciated within academic circles. Additionally, academics have little insight into the full costs and risks associated with drug development. Frequently, negotiations between academia, industry, and funding bodies are stalled in their respective business development and technology transfer departments because negotiations are conducted based on the premise that fair market value is the maximum value one can obtain. Recognition of existing value in which parties are rewarded with milestones and downstream rewards commensurate with success, as well as focus on the societal goals of advancing science and medicine for the benefit of patients, may improve the philosophical stances of all parties as they arrive at the negotiating table. Legislation should also be considered that encourages industry to avail unused IP and shelved new molecular entities (i.e., small molecules newly developed in the drug discovery process) to academic and not-for-profit groups to further advance translation for humanitarian reasons. We believe that it is crucial to strike a fair and equitable compromise between commercial and humanitarian interests.

Material transfer agreements. Differences and lack of communication between academia and industry regarding the development of basic scientific discoveries have also led to significant time delays in academia-industry scientific interactions. Substantial effort and time are spent forming material transfer agreements that hinder scientific advancement. These inefficiencies can be improved by establishing pre-existing umbrella licensing agreements that permit academic-industry scientific collaborations. For example, master agreements have been successfully established between Genentech Inc. and both the University of California and Stanford University and between Kyowa Hakko Kirin and the La Jolla Institute for Allergy and Immunology. These agreements specify how newly generated IP will be assigned as well as how reagents will be shared. In the case of the La Jolla Institute for Allergy and Immunology, the institutional agreement also provides some additional funds to all investigators rather than specific researchers. In these cases, the major obstacles that typically hinder academic-industry alignment have been removed so that academic and industry scientists can focus on the relevant scientific issues. The result is sharing of data on a regular basis and legal negotiations that move forward more quickly, paving a path for more rapid scientific advancements and clinical translation.

\section{Public-private partnerships to coordinate academia- industry collaboration}

Umbrella organizations with specific disease interests have also made efforts to coordinate collaborations between industry and academia. One such new public-private partnership is the recently launched Innovative Medicines Initiative (IMI), a pan-European research and development initiative that is to receive about 2 billion euros in funding over the next 10 years $(7,8)$. The main goals of this venture are to enhance the safety and efficacy of therapies for five disease areas (brain disorders, cancer, metabolic diseases, infectious diseases, and inflammatory diseases) and to remove the bottlenecks in the drug development process. In the United States, the NIH has committed to funding by 2010 a consortium of 60 clinical and translational centers supported $\$ 0.5$ billion annually as an outgrowth of the general clinical research center concept begun in 1959 (8-11).

Non-profit organizations can also serve as facilitators and accelerators to translation (e.g., the Juvenile Diabetes Research Foundation [JDRF] for T1D and the Myelin Repair Foundation for multiple sclerosis). Here, disease-focused organizations have developed strategies to achieve their missions, which range from funding novel basic research proposals to developing academic IP into a marketable product for industry by expending funds directly for industry-sponsored trials and accessory studies. Each 
of these strategies has its advantages and disadvantages. For example, working with industry can accelerate the rational development of drugs because larger teams will be in operation and significant investments from the company will boost development. However, the ability to influence trial design and accessory studies, for example the development of better biomarkers to predict protection or lack thereof from T1D, can frequently be decreased, especially on products at advanced stages of development nearing FDA approval. One issue that should be addressed in most of these types of agreements is that of IP: if a given approach is ultimately abandoned by a company, the IP should be transferred within an acceptable time frame to the funding organization so that the constituency can seek a new licensee and further development. This is necessary because smaller companies in particular might be forced to relinquish certain drugs due to lack of funding and other operational considerations, even if the drugs constitute promising new treatments for autoimmune disorders such as T1D. The Myelin Repair Foundation has set an exemplary path for a collaborative industry-funding body-academia approach. The foundation put into place early on mechanisms to secure IP and fund a handful of academic researchers who are dedicated to translational research projects. Once promising new leads are discovered and, if needed, validated in preclinical models, the Myelin Repair Foundation takes an active role in seeking out licensees and negotiating a rational path for rapid translation.

Conflicts of interest between academia, funding bodies, and industry also arise when time-sensitive industry pressures, particularly for small companies, are pitted against more time-demanding clinical trials for dose selection, mechanism of action studies, and pharmacodynamic analysis. Such time and financial demands can compromise the design of phase II proof-of-concept trials and negatively affect a scientifically promising, but not clinically mature, platform. Could funding agencies, such as the JDRF, play an intermediary role in providing financial support to mitigate the shorter-term financial issues for a longer-term solution and appropriate development of a technology platform? The optimal solution depends on the precise situation and the amount of funds required, but such approaches may provide a bridging mechanism for highrisk novel and time-demanding therapeutic opportunities.

\section{Obstacles to combination therapies for T1D}

There is uniform agreement in the scientific community that combinatorial therapeutic approaches will ultimately be needed to prevent or treat T1D in order to circumvent side effects of single drugs and enhance efficacy of tolerogenic vaccines. Experimental data support this concept (12), but progress has been slow. What are the reasons, and how can we speed things up? Three predominant reasons account for the current delays in testing novel combinatorial therapies for T1D. First, current thinking and traditional drug development schemes require one or both drugs to be approved prior to combination. Second, drug companies frequently shy away from combination therapies because they are afraid that the label of an already licensed drug (for another indication) might become tainted if problems should arise during a combination therapy trial. Last, suitable agreements between companies have to be reached to share IP and licensure. The first two reasons have precluded combination of CD3-specific antibody therapy with oral or nasal insulin (12), while the latter reason has been an obstacle when trying to combine DNA vaccines or glutamic acid decarboxylase (GAD) with systemically acting immune modulators. Changing this situation requires additional discussion with the FDA and novel incentives to companies for making their existing drugs available for T1D combination therapies. The label for an existing drug could be jeopardized if problems arise during a combination therapy trial. Perhaps arrangements could be made to maintain the "harmless" designation of monotherapy with an already-licensed drug in case problems arise with that drug in combination therapy. Last, in order to facilitate agreements between several companies for combination therapies, it would be helpful for funding agencies to provide incentives (such as support of phase I trials and preclinical evaluations) during drug development or consider extending patent lifetimes (9). This would lower the financial exposure for all parties involved. The most profound challenge is to improve the ratio of development cost to market value for therapies for diseases such as T1D, for which insulin substitution provides a therapeutic alternative and sets a well-justified high bar for novel immune-based interventions.

\section{Future directions}

The infrastructure and interest exist to advance translation of a basic understanding of T1D pathogenesis and pathophysiology to improved human health. However, as we have noted, even with a desire and committed resources, additional obstacles remain that hinder more effective translation and bidirectional advancement of science and medicine (see a list of proposed improvements in Suggested changes to scientific and medical advancement processes). Academics frequently have little data at hand to judge the value of a discovery and are driven to oversell new discoveries, unaware of the development time lines and risks associated with drug development. Funding bodies, although prepared to close financial gaps in translating basic science into the clinic, frequently have strong pressure to promise cures by a given date and many times do not have agreements with academics or indus-

\section{A "stealth" team approach to enhancing clinical translation?}

Task force teams should be assembled by funding bodies or private donors with trans-disciplinary expertise in order to facilitate and accelerate clinical translation of basic scientific discoveries. Such teams, which should be small and nimble, would consist of representatives from academia, industry (legal and IP specialists), the NIH and other funding bodies, the FDA, and insurance companies. They should be assembled on a per-case basis and should engage officials from industry and academia in order to facilitate targeted negotiations concerning a particular drug or intervention strategy. They could also provide analytical resources, for example evaluation of the market situation for new drugs for T1D, which would be useful during future negotiations with health insurance providers. Such teams could also be available as a resource for multiple investigators, who might not be familiar with the obstacles they are about to encounter when translating their favorite discovery to clinical use. Last, they should, when necessary, interact with Congress (for example, in conjunction with funding bodies), if major financial or legal obstacles prevent the development of useful therapeutics for a given disease. 
try to recover their own investments should they become abandoned (despite having value) for other reasons. Industry has to meet strict requirements with respect to profitability, which is not always in line with development of drugs for T1D.

Based on the issues discussed in this Personal Perspective, our ideal vision of rapid and optimized cooperation among academia, industry, and funding bodies to find a treatment or cure for T1D is outlined as follows: briefly, it is necessary to first frame an agreement between academic institutions, funding bodies, and industry that will ensure "safe passage" of a new technology, avoid misunderstandings, and align the differential expectations (Figure 1). The ensuing collaboration should serve to verify and extend the initial scientific observation with more preclinical and/or clinical data to support its clinical potential, an endeavor in which funding bodies can help. Industry has the ability to assemble large teams to illuminate a particular approach from many different angles and conduct more extensive studies. During this process, frequent discussions between legal teams and investigators to pursue underlying IP and patents have to occur in order to keep everybody up to date and align the overall approach. Academics should stay involved throughout this process because they are often experts in the area of new discovery and because negative findings abound but rarely get published (funding bodies could build databases of negative findings, for example, through progress reports on their funded grants). Incentives should be provided for the ultimate success of a discovery, not solely for the short-term success of completing a financial transaction to generate money for investors. The early involvement of industry can be very helpful in achieving this. Funding agencies, which would ideally already be involved at an early stage and have precise knowledge of the new intervention and/or technology, could make a commitment to aid with early clinical trials in order to reduce risk and increase incentives for the industry to remain engaged in the T1D field. At this point, a precise understanding of future potential revenues should be reached, possibly involving help from small, specialized teams that would be assembled on a per-case basis (see below and A "stealth" team approach to enhancing clinical translation?). In addition, IP and the right to license could transfer to disease-focused funding agencies such as the JDRF in a reasonable time frame, in case neither industry nor academia is positioned to pursue the approach any further due to financial reasons. Because of their vested interest in find- ing a rapid cure for $\mathrm{T} 1 \mathrm{D}$, such agencies could find new industrial partners to further develop the intervention.

We have defined several hurdles to the efficient translation of basic discoveries into medical practice and proposed potential solutions to overcome these specific hurdles, but how might we further align expectations from each interested party and develop strategies to facilitate translation? We propose an assembly of cooperative, disease-specific task forces $(1,13)$ (see A "stealth" team approach to enhancing clinical translation?) that include stakeholders from academia (both basic research and clinical translation), patient advocacy groups, industry, the NIH, the FDA, and health insurance companies. Such disease-focused teams would be supported by funding organizations such as the JDRF to help forge agreements between companies, help call public attention to issues, accelerate IP and translational progress that can be unnecessarily held up solely for monetary reasons, and ensure that promising translational approaches in T1D are not dropped from the priority list of academics and companies. These teams would be assembled on a per-case basis and would have to be small and flexible. In addition, it would be of interest to create a legal basis for humanitarian obligations: if the intervention at hand has the potential to help patients with T1D, abandoned IP has to be made available without impinging on the rights and interests of the company or university (or other entities) that holds the IP. This should be a national priority.

We have to realize, although novel technology platforms and scientific advances in dissecting the basis for immune responses and their aberrations have positioned us to have great potential in understanding and treating autoimmune diseases such as T1D, major efforts are still required if we are to fully realize the benefits of these technological and scientific advances in human immune-mediated diseases.

\section{Acknowledgments}

The authors thank Eric Brown, Tim Behrens, and Sean Johnston for critical reading of the commentary. M.G. von Herrath is supported by the Brehm coalition and several NIH and JDRF grants.

Address correspondence to: Matthias von Herrath, Center for Type 1 Diabetes Research, La Jolla Institute for Allergy and Immunology, 9420 Athene Circle, La Jolla, California 92037, USA. Phone: (858) 752-6817; E-mail: matthias@liai.org.

\footnotetext{
1. NIH. 2000. Report of the Autoimmune Diseases Coordinating Committee. October 2000. http:// www3.niaid.nih.gov/topics/autoimmune/PDF/ adccrev.pdf.

2. Akinbami, L. 2006. Asthma prevalence, health care use and mortality: United States, 2003-05. http:// www.cdc.gov/nchs/products/pubs/pubd/hestats/ ashtma03-05/asthma03-05.htm.

3. Dantal, J., and Soulillou, J.P. 2005. Immunosuppressive drugs and the risk of cancer after organ transplantation. N. Engl. J. Med. 352:1371-1373.

4. Scandling, J.D., et al. 2008. Tolerance and chimerism after renal and hematopoietic-cell transplantation. N. Engl. J. Med. 358:362-368.

5. DiMasi, J.A., Hansen, R.W., and Grabowski, H.G.
}

2003. The price of innovation: new estimates of drug development costs. J. Health Econ. 22:151-185. 6. Rossini, A.A. 2007. Passing the baton - to whom? J. Clin. Invest. 117:285-288.

7. Innovative Medicines Initiative website. http://imi. europa.eu/index_en.html.

8. Kamel, N., Compton, C., Middelveld, R., Higenbottam, T., and Dahlen, S.E. 2008. The Innovative Medicines Initiative (IMI): a new opportunity for scientific collaboration between academia and industry at the European level. Eur. Respir. J. 31:924-926.

9. National Center for Research Resources. 2009. Clinical and Translational Science Awards. http:// www.ncrr.nih.gov/clinical_research_resources/ clinical_and_translational_science_awards/.
10. United States Food and Drug Administration. 2007. Food and Drug Administration Amendments Act of 2007. http://www.fda.gov/cder/regulatory/FDAAA/default.htm.

11. United States Food and Drug Administration. 2008. Celebrating the successes of the Orphan Drug Act. http://www.fda.gov/consumer/updates/ oda020808.html.

12. Bresson, D., et al. 2006. Anti-CD3 and nasal proinsulin combination therapy enhances remission from recent-onset autoimmune diabetes by inducing Tregs. J. Clin. Invest. 116:1371-1381.

13. NIH Autoimmune Diseases Coordinating Committee. 2002. Autoimmune diseases research plan. http:// www.niaid.nih.gov/dait/pdf/ADCC_Report.pdf. 